\title{
Atwood number effects in buoyancy driven flows
}

\author{
M. J. Andrews \& F. F. Jebrail \\ Los Alamos National Laboratory, USA
}

\begin{abstract}
Consideration is given to Atwood number (non-dimensional density difference) effects in buoyancy driven flows. Buoyancy driven (natural convection) flows may be treated as Boussinesq for small Atwood number, but as Atwood number increases $(>0.1$, i.e. large temperature differences) the Boussinesq approximation is no longer valid and the distinct "bubble" and "spike" geometry of RayleighTaylor buoyant plumes is formed. Aside from asymmetry in the flow the Atwood number also affects key turbulent mix parameters such as the molecular mix, and heat transfer coefficients. This paper will present recent experimental work being performed in the buoyancy driven mix laboratory at Texas A\&M University with air/helium as mixing components. Corresponding numerical simulations performed at Los Alamos are presented for the experiments, and future directions for the research discussed.
\end{abstract}

Keywords: buoyancy, Boussinesq, Atwood number, natural convection.

\section{Introduction}

This paper describes experiments and corresponding simulations to investigate non-Boussinesq effects at high Atwood $(A t)$ number $\left(A t \equiv\left(\rho_{1}-\rho_{2}\right) /\left(\rho_{1}+\rho_{2}\right)\right.$ a non-dimensional density ratio) in buoyancy driven turbulence (Rayleigh-Taylor mixing). The Boussinesq assumption for buoyancy driven flows states that density difference effects need only be accounted for in the gravitational terms, and density may be taken as a constant elsewhere in the equations. At small Atwood number $(A t<<1)$ the Boussinesq approximation is valid, however, at large At $(\sim 1)$ it implies a symmetry to the flow that is contrary to the familiar bubbles and spikes of water falling out of a glass. Here we explore the limits of the Boussinesq approximation. 
An incompressible experiment is described that employs air/helium for $A t=0.035$ (small $A t$ ), and for $A t=0.26$ (high enough to see some asymmetry). Simulations have also been performed on the computer using the MILES (Monotone Integrated LES) method, Youngs [1], and compared with corresponding experiments. Additional simulations have been performed to explore $A t$ effects up to $A t=0.9$. At small $A t$ the buoyancy driven mix develops symmetrically indicating the Boussinesq approximation is valid. However, at high $A t$ number $(>0.1)$ asymmetries in the turbulent mixing become apparent and indicate that the Boussinesq approximation is not appropriate for $A t>0.1$. Consequences for natural convection and high temperature gas heating/cooling problems are discussed.

\section{Experiments}

\subsection{Experimental facility}

A schematic of the experiment is shown in Figure 1. The experimental facility was a wind tunnel, with a splitter plate that separates an upper air stream $\left(\rho_{1}\right)$ from a lower air/helium stream $\left(\rho_{2}\right)$. The stream velocities were kept the same to avoid shear, and great care was taken to obtain a constant and controllable helium flow rate. Thus, by pre-mixing air and helium for the lower stream the density difference between the upper and lower streams could be varied from $A t=0$ (air top and bottom), up to $A t=0.75$ (air on top, and helium on the bottom). More details of the experimental set-up can be found in Banerjee and Andrews [2]. For future reference, the vertical depth of the channel is $1.2 \mathrm{~m}$, and out-of-plane width is $0.6 \mathrm{~m}$. By introducing smoke into the upper channel and using a calibration wedge the density profile can be measured from digital photographs, see Banerjee and Andrews [2].

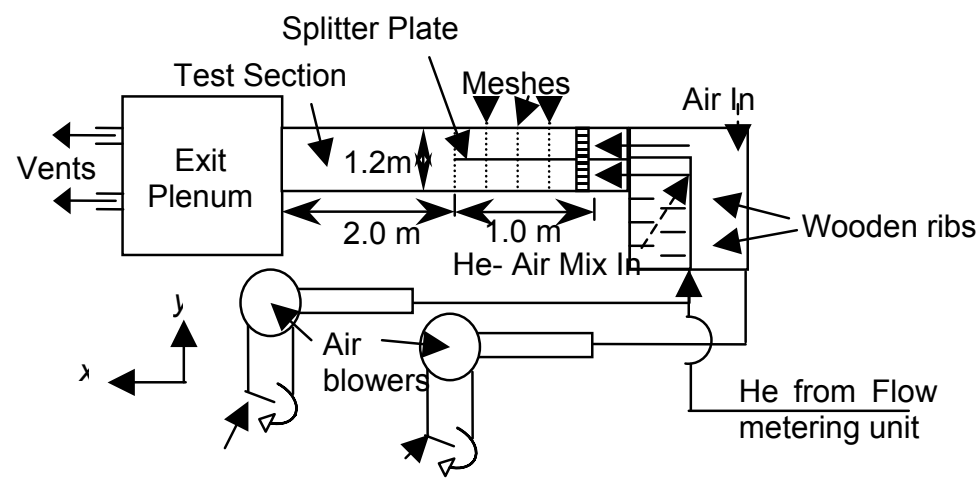

Adjustable dampers

Side View

Figure 1: Schematic of the experimental facility. 


\subsection{Experimental details}

Two experiments are reported in this paper corresponding to $A t=0.035$ and $A t=0.26$. Table 1 reports the experimental conditions (note the stream velocity, $U$, is higher for $A t=0.26$ to keep the spread angle small, and hence a parabolic flow). Use of Taylors' hypothesis relates distance downstream $(x)$ and time $(t)$ as $t=x / U$, and this time is also reported in Table 1 .

Table 1: $\quad$ Experimental parameters.

\begin{tabular}{|c|c|c|c|}
\hline$A t$ & $U(\mathrm{~m} / \mathrm{s})$ & $x(\mathrm{~m})$ & $t(\mathrm{~s})$ \\
\hline 0.035 & 0.6 & 0.75 & 1.25 \\
\hline 0.035 & 0.6 & 1.4 & 2.33 \\
\hline 0.035 & 0.6 & 1.75 & 2.92 \\
\hline 0.26 & 1.2 & 0.5 & 0.42 \\
\hline 0.26 & 1.2 & 1.5 & 1.25 \\
\hline
\end{tabular}

\section{Governing equations and numerical details}

\subsection{Governing equations}

The incompressible Euler equations are used in conjunction with the MILES (see below for more details) modeling technique:

Volume conservation:

$$
\begin{aligned}
& \nabla \bullet \underline{u}=0 \\
& \frac{D f}{D t}=0
\end{aligned}
$$

Scalar transport:

Momentum:

$$
\frac{D(\rho \underline{u})}{D t}=-\nabla p+\rho \underline{g}
$$

with the fluid velocity $\underline{u}=(u, v, w)$, density, $\rho$, pressure, $p$, and gravity, $\underline{g}=\left(0,0, g_{z}\right)$, and scalar $f$. There are six independent variables and five equations, the seventh equation is a linear equation of state for density such as $\rho=L(f)$. In the present work we take $f$ to be the non-dimensional density, or mixture fraction, defined as $f=\left(\rho-\rho_{2}\right) /\left(\rho_{1}-\rho_{2}\right)$.

\subsection{Numerical solution procedure}

\subsubsection{Overview}

For the present experiment we have used MILES, namely, Monotone Integrated LES. MILES modeling involves solving Euler governing equations and using numerical diffusion to model turbulent diffusion. Success with this modeling technique for buoyancy driven flows has been reported by Youngs [1]. 
The governing equations presented above are a coupled set of partial differential equations for which there exist several solution procedures. The present work solves the governing equations using the RTI3D code described by Andrews [3]. In particular, a fractional time step technique is used in which for each time step an advection calculation is followed by a Lagrangian source term update. The Lagrangian update is presented next, and this is followed by a brief description of the advection step for the scalar $f$ (details may be found in Andrews [3]).

\subsubsection{Lagrangian momentum source term updates}

The Lagrangian $w$ momentum equation is:

$$
w_{n}^{*}=w_{n}^{n+1 / 2}+\frac{\Delta t}{\rho_{t} \Delta y}\left(p_{P}^{n}-p_{T}^{n}\right)+g_{z}
$$

The $n+1 / 2$ superscript refers to a value from the advection calculation, and * to an intermediate value that does not necessarily satisfy continuity. The subscripts refer to spatial position (north face), typical of the SIMPLE method (Patankar [4]), and a staggered arrangement of momentum and mass cells is used. Following the SIMPLE practice, velocity corrections are defined so that $u_{i, e}^{n+1}=u_{i, e}^{*}+\Delta u_{i, e}$ (and similarly for the other velocities) and a new pressure $p_{P}^{n+1}=p_{P}^{n}+\Delta p_{p}$ where $\Delta p$ is a pressure correction. By substituting these expressions for $n+1$ into the volume conservation equation and then subtracting equation (4) evaluated with the * we arrive at the usual Poisson equation for pressure corrections:

$$
a_{P} \Delta p_{P}+a_{E} \Delta p_{E}+a_{W} \Delta p_{W}+a_{N} \Delta p_{N}+a_{S} \Delta p_{S}=-D i v
$$

with Div the divergence of the * velocity values. The Poisson equation (5) is solved using a Full Multi-Grid method, and the pressure corrections are used in a SIMPLE style to provide updated $n+1$ velocities and pressures that simultaneously satisfy the momentum equations (3) and volume conservation.

\subsubsection{Transport procedures}

The 3D transport procedures are split into $x / y / z$-steps, this fractional splitting simplifies the calculation to one-dimensional updates that lends itself to high order calculation of cell fluxes with the Van Leer [5] method. There follows a brief description of the scalar $x$-step advection, the $y$ and $z$ steps being similar, and similar advection steps are performed for the momentum.

The $x$-step advection for the scalar is given by:

$$
f_{P}^{*}=f_{P}^{n}+\Delta y \Delta z \Delta t\left(u_{e} f_{e}-u_{w} f_{w}\right)
$$

where $P$ refers to the center of a control volume, $e$ the east face, and $w$ the west face. The face values for the $u$ velocities are available, and the face values for the scalar are computed using a second order approximation with Van-Leer limiting to prevent non-physical oscillation as: 


$$
f_{e}=f_{\text {upwind }}+\operatorname{sign}\left(\varepsilon_{e}\right) \frac{\left(1-\varepsilon_{e}\right)}{2} \Delta x D_{e}
$$

where $\varepsilon_{e}=\Delta t u_{e} / \Delta x$, and upwind values are taken according to the sign of $\varepsilon_{e}$. The derivative is evaluated following Van Leer as:

$$
D_{e}=S \min \left\{|D|, \quad \frac{2\left|\Delta_{w}\right|}{\Delta x}, \quad \frac{2\left|\Delta_{e}\right|}{\Delta x}\right\}
$$

where $\Delta_{w}=f_{P}^{n}-f_{W}^{n}, \Delta_{e}=f_{E}^{n}-f_{P}^{n}$ and $S=\left\{\begin{array}{cc}1 & \text { if } \Delta_{e} \text { and } \Delta_{w}>0 \\ -1 & \text { if } \Delta_{e} \text { and } \Delta_{w}<0 \\ 0 & \text { otherwise }\end{array}\right.$

Van Leer limiters have been used in equation (8) to limit the gradient of the volume fraction profile, thereby preventing spurious oscillations. The representation for the gradient of the cell profile $D$ determines the accuracy of the representation.

In the present work $D=\left(\Delta_{e}+\Delta_{w}\right) /(2 \Delta x)$, so the gradient is computed with a central difference so this scheme is referred to as "2nd order".

\subsection{Computational details for simulation of the experiments}

The experiments are performed in a statistically steady gas channel, but are modelled on the computer using transient simulations that are related to the experiment through the Taylor hypothesis described above. The computational domain is taken to be $1.2 \mathrm{~m}$ high $(z)$, and $0.6 \mathrm{~m}$ square $(x \& y)$, with a computational grid of $64 \times 64 \times 128(x-y-z)$. The computational time step is selected by the computer program to keep the Courant number below 0.25 . Initial conditions for the simulations are prescribed to fit the density interface off the splitter plate. Here we use the following initial density interface perturbations:

$$
\begin{array}{r}
h(x, y)=h_{w}+\sum_{k_{x}, k_{y}} a_{k} \cos \left(k_{x} x\right) \cos \left(k_{y} y\right)+b_{k} \cos \left(k_{x} x\right) \sin \left(k_{y} y\right)+ \\
c_{k} \sin \left(k_{x} x\right) \cos \left(k_{y} y\right)+d_{k} \sin \left(k_{x} x\right) \sin \left(k_{y} y\right)
\end{array}
$$

where the spectral amplitudes are chosen randomly but give an rms amplitude of $0.6 \mathrm{~m} / 100$, and the wave numbers range from modes 8 to 16 (Dimonte et al. [6]). The wake off the splitter plate is modelled as an initial perturbation, $h_{w}$, where $h_{w}=a_{w} \sin \left(k_{w} x\right) \cos \left(k_{w} y\right)$; the wavelength associated with the wave number $k_{w}$ is $1 \mathrm{~cm}$ and is taken from inspection of the experimental photograph in Figure 2, and the amplitude is $0.5 \mathrm{~cm}$, again taken from Figure 2.

\section{Results and discussion}

Figure 2 is a photograph taken from the $A t=0.26$ experiment. On the right at the mid-plane is the splitter plate. The air/helium streams move from right to left, and the buoyancy driven mix is seen to develop downstream. On the far left of 
the photograph ( $2 \mathrm{~m}$ from the splitter plate) the mixing has expanded to the top and bottom of the channel (i.e. a total depth of $120 \mathrm{~cm}$ ). The photograph shows a well mixed region with a wide range of mixing length scales. Close inspection of the region close to the end of the splitter plate, reveals initial perturbations of wavelength about $1 \mathrm{~cm}$, and amplitude $0.5 \mathrm{~cm}$, associated with wake shedding.

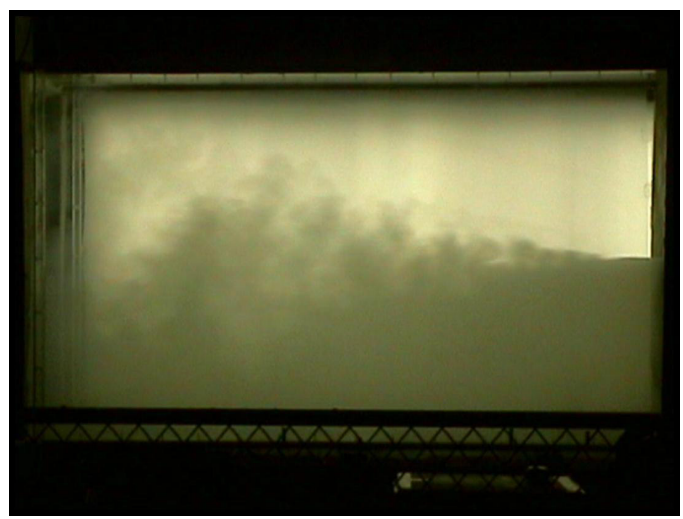

Figure 2: $\quad$ Photograph from $A t=0.26$ experiment.

Figure 3 below shows plots of initial density interface (on the left) used for the $A t=0.035$ simulations (and is the same for all the simulations), and it is evident that there are both short and longer wavelength disturbances corresponding to conditions at the end of the splitter plate. The right side of Figure 3 shows the mix edges interfaces at $f=0.01$ (lower surface) and $f=0.99$ (upper surface), and corresponds to a distance downstream of $175 \mathrm{~cm}$ (near the left side of the photograph in Figure 2). Comparison of the computed and experimental disturbances in Figure 2 and the right of Figure 3 shows more fine scale in the experiment, and this is because of computational grid resolution.
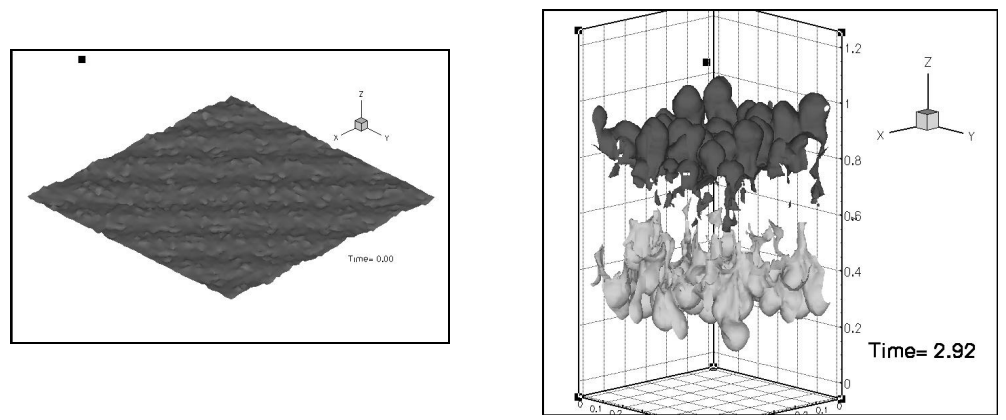

Figure 3: Initial conditions and late time $(t=2.92 \mathrm{~s})$ mixing edges for $A t=0.035$. 
Calibration of digital photographs provided time averaged non-dimensional density profiles (the scalar fraction, $f$ ) and these are shown in Figures 4 and 5, with the corresponding results from the simulations using MILES. Comparison in Figure 4 for an $A t=0.035$ of the experimental density widths and profiles with the corresponding simulations is good, and in particular the almost linear density profile across the mixing region is well captured by the MILES simulations. Also of note is that the experimental mix is symmetric around the centreline, indicating that the Boussinesq approximation is valid for an $A t=0.035$. Similarly, in Figure 5, comparison of experiment with simulation for the $A t=0.26$ show they also agree quite well. However, the experiments and the simulations show a slight asymmetry, associated with the higher $A t=0.26$, that perhaps best seen by inspecting the penetration at the edges of the mix in Figure 5.
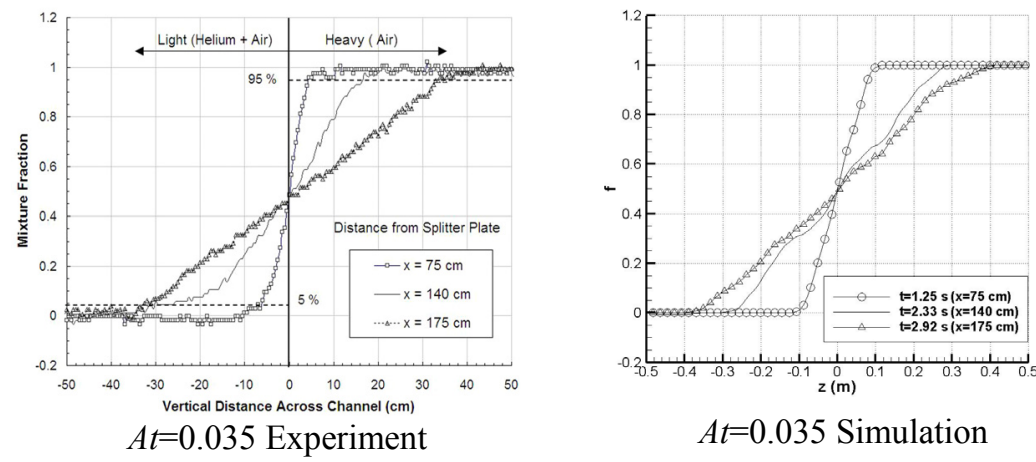

Figure 4: Comparison of mix profiles for $A t=0.035$.
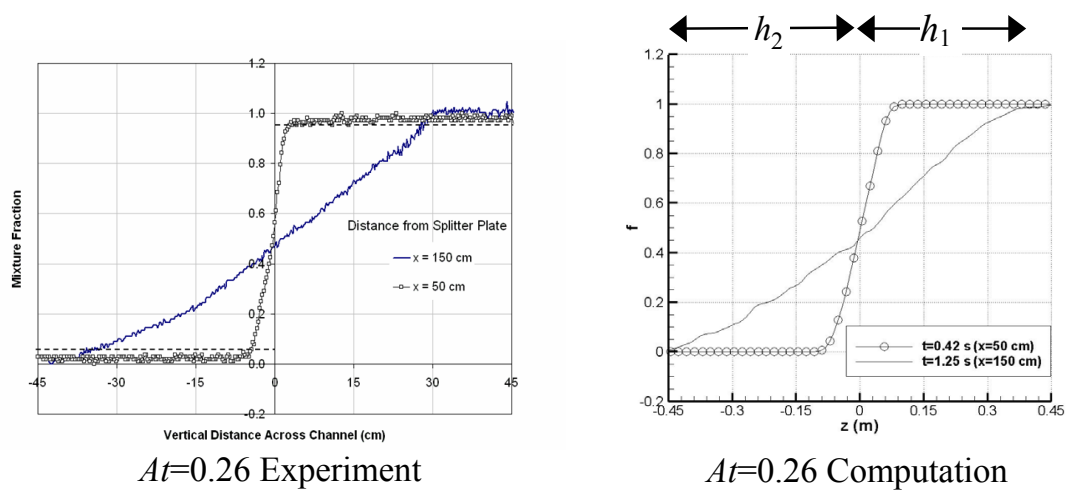

Figure 5: $\quad$ Comparison of mix profiles for $A t=0.26$.

To explore higher At (i.e. large density differences) two additional simulations have been performed for $A t=0.5$ and $A t=0.9$. To facilitate comparison, the product $A t^{*} g_{z}$ was held constant at the value for the $A t=0.035$ 
case, so $A t^{*} g_{z}=-0.34335 \mathrm{~ms}^{-2}$, and the value of $g_{z}$ then computed for each value of $A t$, so for $A t=0.5$ a value of $g_{z}=-0.6867 \mathrm{~ms}^{-2}$ was assigned, and for $A t=0.9$ the value was $g_{\mathrm{z}}=-0.3815 \mathrm{~ms}^{-2}$. This scaling comes from consideration of the gravitational term in the governing equations. To gauge asymmetry the bubble penetration, $h_{1}$, and spike penetration, $h_{2}$, were computed as the distance from the centreline to the values of $f=0.99$ and $f=0.01$ respectively (see Figure 5 for a graphical representation at $t=1.25 \mathrm{~s}$ ), and their ratio is plotted in Figure 6 . Inspection of Figure 6 reveals that there is practically no asymmetry for $A t=0.035$, however, asymmetry is clearly seen for $A t=0.5$, with the spike/bubble penetration being about 1.2 near the end of the calculation at $t=2 \mathrm{~s}$. Indeed, there is a strong asymmetry for $A t=0.9$ with $h_{2} / h_{l}=1.7$ by $t=2 \mathrm{~s}$. Figure 7 plots the edges of the mix region, in a similar fashion to Figure 5 , for each of the cases at $t=2 \mathrm{~s}$. The $A t^{*} g_{z}$ scaling ensures a comparison at similar development of the mix. Comparison of the mix edges for $A t=0.035$ with $A t=0.5$ in Figure 7 shows little difference in bubble and spike penetration. However, for $A t=0.9$ the asymmetry is clear, with bubbles clearly shown at the top, and finger shaped spikes at the bottom.

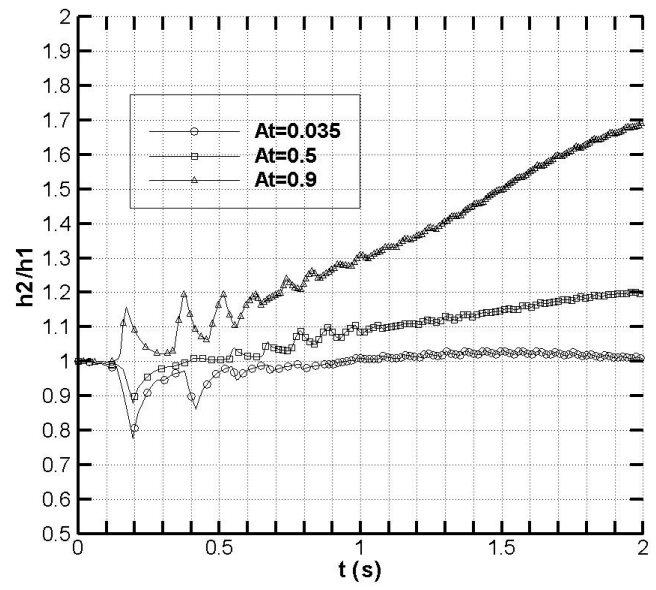

Figure 6: $\quad$ Spike to bubble penetration $\left(h_{2} / h_{1}\right)$ asymmetry for increasing $A t$

Results from the simulations show that significant departures from the Boussinesq approximation occur by $A t=0.5$, but are not particularly evident at $A t=0.26$. This suggests that the Boussinesq approximation is reasonable for pure buoyancy flows at least for $A t<0.1$, and perhaps as high as $A t<0.3$. In terms of hot/cold gas flows, for an ideal gas at constant pressure the density ratio $\rho_{1} / \rho_{2}$ corresponds to a temperature ratio of cold to hot, and for $A t=0.1$ the density ratio is $1.22 / 1$, or temperature ratio of $1000 \mathrm{~K} / 1220 \mathrm{~K}$; for $A t=0.3$ the density ratio is $1.86 / 1$, or $1000 \mathrm{~K} / 1860 \mathrm{~K}$. Thus, it would appear for many free convection problems the Boussinesq approximation would be valid. However, for 
combustion problems and other problems associated with large temperature differences, the full set of equations should be solved when in the presence of a body force (e.g. gravity or centrifugal).

We close with a few words of caution - for accurate comparison of experiment and simulation we need better characterization of the initial conditions, i.e., the initial spectrum being shed from the splitter plate. In addition, our use of a $64 \times 64 \times 128$ grid should be considered a coarse resolution, and additional calculations should be performed for higher resolution to confirm the results.

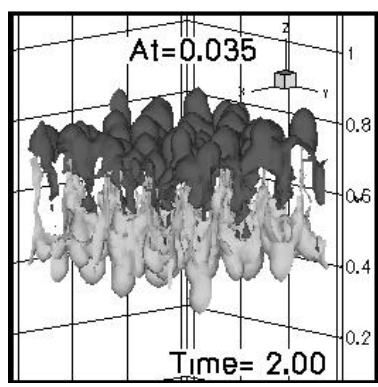

Figure 7: $\quad$ Mix edges as a function of $A t$.

\section{Conclusions}

A buoyancy driven mix experiment has been described, with corresponding simulations on the computer. Results from experiments at Atwood (At) numbers of 0.035 and 0.026 have been compared with corresponding MILES simulations and found satisfactory. Additional simulations at high At of 0.5 and 0.9 reveal a strong asymmetry in the buoyancy driven mix that indicates the Boussinesq approximation is no longer valid. However, the results suggest that the Boussinesq approximation is valid at least for $A t<0.1$, and perhaps as high as $A t<0.3$. Additional work is required to match the initial conditions of the experiment, and to perform additional simulations with higher grid resolutions to provide further refinement on the $A t$ criteria proposed.

\section{Acknowledgements}

This paper is dedicated to the memory of Sara Boyce Andrews (1926-2006).

\section{References}

[1] Youngs, D.L., "Application of MILES to Rayleigh-Taylor and RichtmeyerMeshkov mixing", 16th AIAA Computational Fluid Dynamics Conference, 23-26 June 2003 AIAA 2003-4102. 
[2] Banerjee, A., and Andrews, M.J., "A Gas Channel Facility to Investigate Statistically Steady Rayleigh-Taylor Mixing at High Atwood Numbers," accepted to Physics of Fluids, December, 2006.

[3] Andrews, M.J., "Accurate Computation of Convective Transport in Transient Two-Phase Flow," International Journal for Numerical Methods in Fluids, Vol. 21, No. 3, pp. 205-222, 1995.

[4] Patankar, S.V., "Numerical Heat Transfer and Fluid Flow", Hemisphere, 1980.

[5] Van Leer, B., "Towards the Ultimate Conservative Difference Scheme, IV. A new Approach to Numerical Convection", J. Comp. Phys., Vol 23, pp 276-299, 1977.

[6] Dimonte, G., Youngs, D.L., Dimits, A., Weber, S., Marinak, M., Calder, A.C., Fryxell, B., Biello, J., Dursi, L., MacNeice, P., Olson, K., Ricker, P., Rosner, R., Timmes, F., Tufo, H., Youns, Y.-N., Zingale, M., Wunsch, S., Garasi, C., Robinson, A., Ramaprabhu, P., and Andrews, M.J., "A Comparative Study of the Turbulent Rayleigh-Taylor (RT) Instability Using High-Resolution 3D Numerical Simulations: The Alpha Group Collaboration," Physics of Fluids A, Vol. 16, No. 5, pp. 1668-1693, May 2004. 